\title{
Rapid Infrared Microspectroscopy using an Infrared Spectrograph Based on a Prism Dispersing Element and an MCT Array Detector.
}

\author{
Zachary Keltner*, Katherine Kayima*, Luis Lavalle*, Marina Canepa*, Anthony E. Dowrey**, \\ Gloria M. Story**, Curtis Marcott** and Andre' J. Sommer* \\ *Molecular Microspectroscopy Laboratory, Department of Chemistry and Biochemistry, \\ Miami University, Oxford, OH 45056. \\ **The Procter and Gamble Company, Miami Valley Innovation Center, Cincinnati, Ohio 45253.
}

Over the last two decades infrared microspectroscopy has been employed in a wide variety of disciplines to elucidate chemical information from small spatial domains. Although this method is mature from an instrumental standpoint, one drawback is the method's temporal resolution. Measurements in the mid-infrared spectral region continue to be dominated by interferometers whose temporal resolution is directly tied to the modulation efficiency of the detector. For example, a typical mercury cadmium telluride (MCT) detector operates best at an optical path difference velocity of $1-2 \mathrm{~cm} / \mathrm{sec}$. If $4 \mathrm{~cm}^{-1}$ resolution is required, the fastest spectral acquisition rate would be one spectrum every 250-125 milliseconds.

The recent availability of array detectors has led researchers to investigate spectrographs using these detectors as a means to increase the temporal resolution in infrared spectroscopy [1-7] . Although array detectors have been available for the past 16 years the overall concept is not new. In 1951 Agnew, Franklin and Ben constructed a prism-based spectrograph using 10 Golay detectors [8]. The output from the detectors was coupled to an oscilloscope such that real-time data could be observed from 10 different wavelengths. More recently, Richardson et al. began to investigate the use of a modern-day InSb array detector in a grating based spectrograph. They reported the ability to collect a spectrum in 4 microseconds at $32 \mathrm{~cm}^{-1}$ resolution with a signal to noise ratio (SNR) of 50:1 [1]. They later reported on an improved instrument, which afforded a higher spectral resolution and SNR while compromising the temporal resolution slightly [2]. Finally, Elmore et al. and Snively et al. reported on grating based spectrographs using current InSb and MCT technology [4-6]. These latest advances allowed the method to be applied to diverse systems including monolayer films [5] and rheo-optical studies of polymers [7].

In our preliminary investigations using a grating based spectrograph and MCT array we found that the dispersion of the grating and the array size did not complement one another. The high dispersion of the grating yielded high resolution but the array size limited the spectral range to a few hundred wavenumbers. Snively increased the spectral range slightly by using an infrared camera lens affixed to the detector, albeit at increased expense to the system [6]. Due to these difficulties we decided to explore the use of prisms as dispersing elements which characteristically have lower dispersion.

A spectrograph was constructed as shown in Figure 1 using a silicon carbide source, $150 \mathrm{~mm}$ focal length source optics, a $300 \mathrm{~mm}$ focal length focusing mirror, a sodium chloride prism and a 256 element MCT focal plane array detector. A spectrum of polystyrene shown in Figure 2 illustrates coverage from $\sim 3600$ to $1200 \mathrm{~cm}^{-1}$ with an estimated spectral resolution of slightly 
less than $16 \mathrm{~cm}^{-1}$. This spectrum was collected in $\sim 10$ seconds and is the average of $\sim 100$ spectra down the height of the array.

In light of the success of this arrangement we decided to interface the spectrograph to an IRPLAN microscope. The typical interface box of the IRPLAN was removed and a $150 \mathrm{~mm}$ focal length mirror was used to collimate the source for input into the microscope. The output of the microscope was brought to a focus using a $200 \mathrm{~mm}$ focal length lens and re-collimated using a $150 \mathrm{~mm}$ focal lens for direct input into the prism. The microscope's aperture located after the sample served as the entrance slit for the spectrograph and the sample's area defining aperture. Figure 2 illustrates an infrared spectrum obtained on a polystyrene sample under the same conditions as above using a slit size corresponding to a 10 micrometer sample. A comparison of this spectrum to that of the macro setup, shows similar coverage but an apparent loss in resolution. This result is expected due to the fact that the interface between the microscope and the spectrograph has not been optimized. Finally, although the spectra were collected in 10 seconds, real time images of the detector output clearly showed bands associated with the polystyrene at a frame rate of 17 milliseconds.

[1] H. H. Richardson, V. W. Pabst and J. A. Butcher, Appl. Spectrosc., 44, 822 (1990).

[2] H. H. Richardson, V. W. Pabst and J. A. Butcher, Appl. Spectrosc., 47, 1626 (1993).

[3] P. Hamm, S. Wiemann, M. Zurek and W. Zinth, Opt. Lett., 19, 1642 (1994).

[4] D. L. Elmore, et al., Appl. Spectrosc., 56, 145 (2002).

[5] D. L. Elmore, et al., Langmuir, 19, 3519, (2003).

[6] C. M. Snively, C. Pellerin, J. F. Rabolt and D. B. Chase, Anal. Chem., 76, 1811, (2004).

[7] C. Pellerin, S. Frisk, J. F. Rabolt, D.B. Chase, Appl. Spectros., 58, 799 (2004).

[8] J. T. Agnew, R. G. Franklin and R. E. Benn, J. Opt. Soc. Amer., 41, 76, (1951).
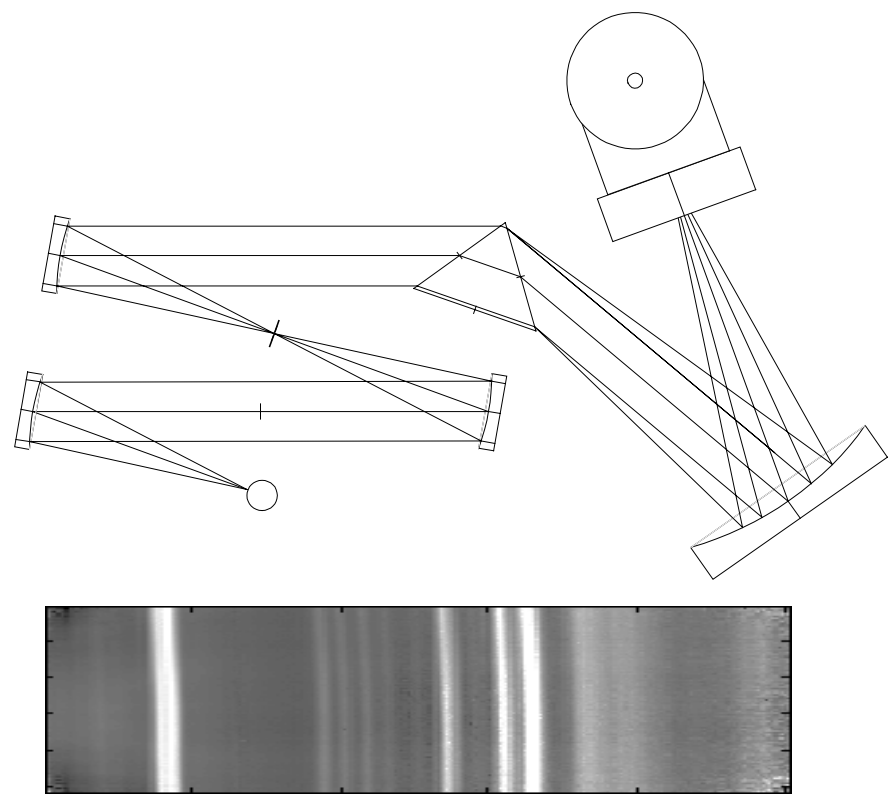

Figure 1. Optical diagram of the prism spectrograph (top) and real-time image of detector output (bottom).

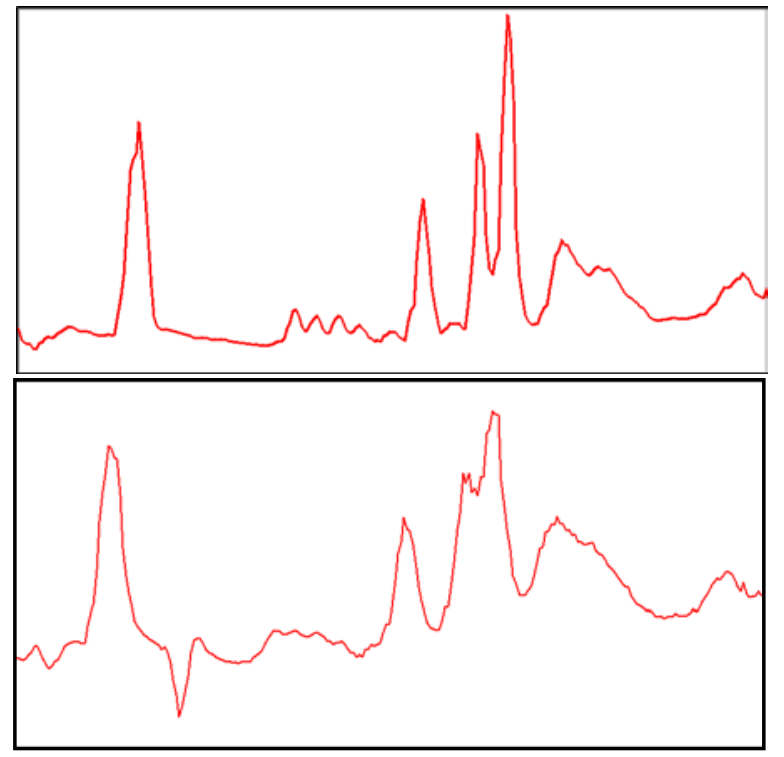

Figure 2. Macro infrared spectrum of polystyrene (top) and micro infrared spectrum of polystyrene (bottom). 
https://doi.org/10.1017/S1431927606066633 Published online by Cambridge University Press 